\title{
Performance Analysis of Dynamic Source Routing Protocol in TCP and UDP Environment in MANET Varying Number of Nodes
}

\author{
Neelpawan Kalita \\ M.Tech CSE \\ Girijananda Chowdhury Institute of Management \\ and Technology \\ Guwahati, Assam, India
}

\author{
Utpal Barman \\ Asst. Professor \\ Girijananda Chowdhury Institute of Management \\ and Technology \\ Guwahati, Assam, India
}

\begin{abstract}
Dynamic Source Routing (DSR) is a proficient on-interest routing protocol for mobile ad-hoc networks (MANET). It relies on upon two fundamental systems: Route Discovery and Route Maintenance. Route discovery is the methodology utilized at the source of the packets to find a route to the destination. Route Maintenance is the methodology that finds link breakage also, repairs them. Route caching is the sub method serviceable to keep away from the interest for finding a route or to decrease route discovery delay before each information bundle is sent. The objective of this paper is to assess the execution of DSR in TCP and UDP environment. Distinctive execution expressions are explored including, average throughput, end to-end delay, and average packet delivery ratio, depending on TCP and UDP environment by varying number of nodes. In this study we find which environment best suit for DSR protocol.
\end{abstract}

\section{Keywords}

MANET, NS-2, Routing, DSR, TCP, UDP

\section{INTRODUCTION}

A Mobile Ad-hoc Network (MANET) is the dynamic examination subject in remote interchanges. It is a accumulation of two or more hubs in which the correspondence connections are remote; without the administration of any settled framework or incorporated director. The system is Ad-hoc on the grounds that every hub can get what's more, forward information to different hubs, thus the judgment of which hubs forward information is made progressively in light of the system availability. The benefit of this sort of system is that it doesn't require any sort of infrastructure, similar to a base station in cell system, so the most imperative test in a mobile Ad-hoc network is to execute routing protocols that can deal with these network topology changes to maintain up and rebuild dependable routes in a timely approach [1].

\section{ROUTING}

A few different routing algorithm for Ad-hoc systems, with their specific points of and disservices have been proposed as of not long ago. Scientists customarily arrange these conventions as proactive conventions, receptive conventions, or half and half of them, in light of the calculations that discover new courses or redesign existing ones. Proactive directing is actualized by trading steering tables, (for example, SDV, WRP). Responsive steering is on interest steering, (for example, DSR, AODV). It has been demonstrated that responsive steering is more suited for Ad-hoc than the proactive one. In responsive steering, there is two fundamental stages: Route Discovery and route maintenance [1]. The route discovery stage depends on solicitation and answer reuses, so the expense is high, which will diminish the execution of system. The examiners utilize three essential procedures to abatement disclosure cost: - Enhancing reserve, each hub has a neighbourhood store to spare the defeat way from itself to destination, (for example, DSR, AODV). - Local flooding, use show flooding to particular neighbours in view of particular controls. The flooding telecast locally will diminish the revelation cost, (for example, LAR, ZRP) [9]. Multiple course way, utilizing various ways to transmit information parallel or simultaneously, alternative way will decrease the quantity of solicitations, (for example, SMR, AOMDV) [9]. On-interest directing conventions accomplish superior to anything table driven directing conventions in versatile Ad-hoc systems. In an on-interest technique, hub endeavours to find a trustworthy course when it needs send information bundles to a destination. To maintain a strategic distance from the expense of finding a course for every information bundle, hubs store that found routes in a reserve. Therefore, a viable reserving procedure is a crucial part of any on-interest directing convention for remote Adhoc systems to stay up with the latest dependably [2].

\section{LITERATURE REVIEW}

In this paper, we have discussed the various approaches present for Routing protocols in MANET. Here we have discussed DSR Routing protocol in MANET. Some of the important literatures which are considered more important survey for our project are discussed below.

Amer O. Abu Salem et al. explained that the DSR routing protocol has acceptable performance in terms of data packet delivery ratio, throughput and they focused on varying the cache size and the speed by simulation using NS-2.[1]

Salman bhimla et al. has said that for a high mobile network, when queue size is very less, the packets are dropped and buffer over flows for DSR protocol. Also the network load increases and through will also increases as compared to the high queue size for high mobile network.[2]

Amit N. Thakare et al. said that DSR is more stable and has less overhead. DSR can make use of multiple path and does not send a periodic packet. Moreover it stores all usable routing information extracted from overhearing packets. However, these overheard route information could lead to inconsistencies.[3]

Zaiba Ishrat, et al. said that DSR perform better PDR, DELAY and THROUGHPUT and the performance of ZRP is good for less number of nodes and its performance decreases 
when number of nodes increases. When the pause time is less throughput is low for DSR. Simulation results show that better performance is achieved in DSR protocol in terms of packet loss, throughput over a discontinuous network.[4].

Utpal Barman, et al. said that in AODV routing protocol increasing the number of nodes the throughput also increases but packet delivery ratio decreases [10].

Utpal Barman et al. explained that in DSDV routing protocol throughput is better with the increase of number of node again a delay is less. But it is not possible in case of node mobility [11].

\section{DESCRIPTION OF REACTIVE PROTOCOL}

Relative protocol is recognized as On-demand protocol on the grounds that it makes connects just when these connections are required. The need is started by the source, as the name proposes. At the point when a source hub requires a route to a destination, it starts a route discovery process inside of the system. This procedure is finished once a route is discovered. After that there is a route upkeep technique to keep up the substantial routes and to evacuate the invalid routes [1].

\subsection{Dynamic Source Routing (Dsr)}

Dynamic Source Routing (DSR) is a ROUTING PROTOCOL for remote network systems. It is like AODV in that it builds up a course on-interest when a transmitting versatile hub demands one. Be that as it may, it utilizes source directing as opposed to depending on the ROUTING table at every middle of the road gadget. Dynamic source routing protocol (DSR) is an on-interest, source routing protocol, whereby all the directing data is kept up (ceaselessly upgraded) at portable hubs. DSR permits the system to be totally self-sorting out and self-arranging, without the requirement for any current system foundation or organization. The convention is made out of the two primary components of "Route Discovery" and "Route Maintenance", which cooperate to permit hubs to find and keep up routes to discretionary destinations in the specially appointed system [3]. An ideal way for a correspondence between a source hub and target hub is dictated by Route Discovery process. Route Upkeep guarantees that the correspondence way remains ideal and circle free concurring the adjustment in system conditions, regardless of the fact that this requires adjusting the route amid a transmission. Route Reply would just be produced if the message has achieved the anticipated destination hub (course record which is firstly contained in Route Request would be embedded into the route Reply) [3]. To give back the Route Reply, the destination hub must have a route to the source hub. In the event that the route is in the route store of target hub, the route would be utilized. Something else, the hub will turn around the route in view of the route record in the Route Reply message header (symmetric connections). In case of lethal transmission, the Route Maintenance Phase is started whereby the Route Error packets are produced at a hub. The off base jump will be separated from the hub's route store; all routes containing the jump are decreased by then. Once more, the Route Discovery Phase is started to decide the most feasible course. The real disparity amongst this and the other on-interest routing protocol is that it is reference point less and henceforth it doesn't have need of intermittent hi packets (reference point) transmissions, which are utilized by a hub to advise its neighbours of its nearness. The central methodology of this protocol amid the route creation stage is to dispatch a route by flooding Route Request parcels in the system. The destination hub, on getting a Route Request packet, reacts by exchanging a Route Reply bundle back to the source, which conveys the route crossed by the Route Request packet got [1].

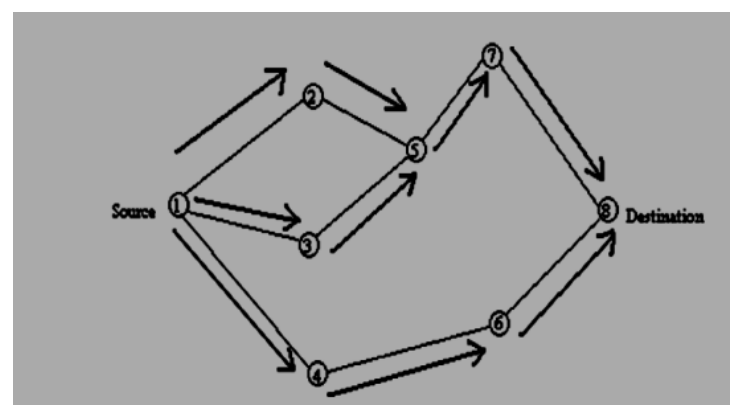

Fig 1. (a) Propagation of RREQ packet [1]

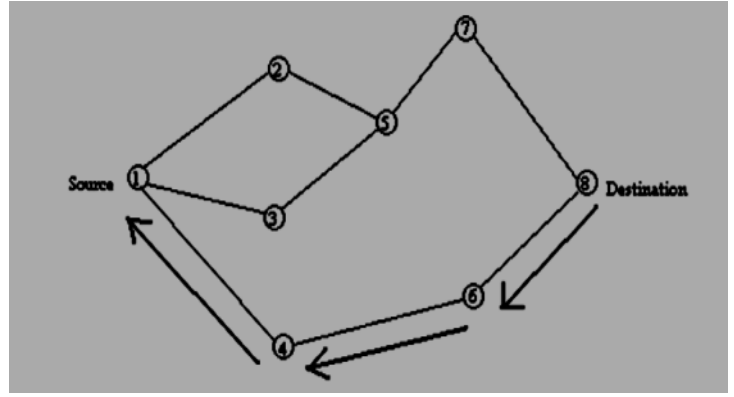

(b) Route creation in DSR [1]

\subsection{CACHING}

A cache system in reactive protocol is characterized by the accompanying arrangement of outline issues that decide store administration technique; the first issue is a store strategy; which it is the arrangement of standards that decide the reserve data structure, to be suited to the route reserve on interest. The specialists proposed and examined two distinctive store structures, called way reserve and connection reserve, and connected them to DSR [5]. A way store is organized that every reserve passage acts a full way from a source to all destinations, while a connection reserve has each singular connection to one destination. Thus, a connection reserve has the conceivable to utilize the course data all the more effectively. The second issue is a perused approach; the principles that decide at the point when utilize a reserve section, and choose what is the response at the point when sending another message from the source hub. For illustration, DSR convention relies on upon a few arrangements: store replay, to answer message with data around a course ask for that put away in store of middle of the road hubs, the second approach is a rescuing answer, when information bundle meet a softened connection up the course way, the moderate hub can utilize elective way to destination from its own store, at last, volunteer answer, a hub can listens for bundles not coordinated to it (packet sniffing), if the hub has a superior route to the destination hub of a bundle, it sends a message answer to the source hub with this new better route [3]. The third issue is a written work arrangement; the guidelines for deciding when to compose a section into the reserve, and which data must be reserved. The principle inconvenience for the written work arrangement is assurance to reserve legitimate ways. The last one is a cancellation arrangement; it is the guidelines for deciding when to erase a section from the reserve and which data must be erased from the reserve. Erasure approach is truly the most basic part of a reserve plan. Two sorts of "mistakes" can happen: Early erasure, a reserved 
course is erased when it is still legitimate. Furthermore, late erasure, a stored route is not erased regardless of the fact that it is no more substantial. As DSR store depends on way association methodology, which rely on upon get course answer packets by halfway or source hub to reserve this way with no handling, and it will erase entire way at the point when an information parcel meets a broken connection by accepting blunder packets, and there is no programmed join close technique. These will come about Inefficient Cache Organization [3].

\subsection{Transmission Control Protocol (Tep) And User Datagram Protocol (Udp)}

The Transmission Control Protocol (TCP) is a center convention of the Internet protocol suite. It started in the underlying system usage in which it supplemented the Internet Protocol (IP). In this manner, the whole suite is normally alluded to as TCP/IP. TCP gives dependable, requested, and blunder checked conveyance of a flood of octets between applications running on hosts imparting over an IP system. Real Internet applications, for example, the World Wide Web, email, remote organization and record exchange depend on TCP. Applications that don't require dependable information stream administration may utilize the User Datagram Protocol (UDP), which gives a connectionless datagram administration that underscores decreased dormancy over dependability.

Then again UDP utilizes a straightforward connectionless transmission model with at least convention instrument. It has no handshaking exchanges, and subsequently uncovered the client's system to any lack of quality of the fundamental system convention. There is no certification of conveyance, requesting, or copy security. UDP gives checksums to information respectability, and port numbers for tending to various capacities at the source and destination of the datagram.

With UDP, PC applications can send messages, for this situation alluded to as datagram's, to different hosts on an Internet Protocol (IP) system without earlier correspondences to set up uncommon transmission channels or information ways. UDP is reasonable for purposes where mistake checking and adjustment is either a bit much or is performed in the application, keeping away from the overhead of such preparing at the system interface level. Time-touchy applications frequently utilize UDP on the grounds that dropping parcels is desirable over sitting tight for deferred bundles, which may not be a choice in a constant framework. On the off chance that blunder remedy offices are required at the system interface level, an application may utilize the Transmission Control Protocol (TCP) or Stream Control Transmission Protocol (SCTP) which are intended for this reason [11].

\section{PERFORMANCE METRICS}

In DSR, we are trying to analyze the performance of this protocol in two different environments i.e. TCP and UDP by varying number of nodes. in this study we tried to analyze DSR protocol best suits in which environment TCP or UDP. Here we analyze End-to-End Delay, Throughput and Packet Delivery Ratio.

\subsection{Average End-to-End Delay}

This is the average time a data packet takes to access the destination. This metric is calculated as: the time at which first data packet arrived to destination. The time at which first packet was transmitted by source. This includes all possible delays caused by buffering for route discovery, queuing at interface queue, retransmission delays at MAC, propagation and transfer time [1].

\subsection{Average Throughput}

Average Throughput (messages/second) is the average rate of successful packet delivery data packets divided over a communication channel, this metric is calculated as:

The average total number of delivered packets divided by the total duration of simulation time. We analyze the throughput in TCP and UDP by varying number of nodes.

\subsection{Data packet delivery ratio}

Total number of delivered data packets divided by total number of data packets transmitted by all nodes [1]

\section{RESULT ANALYSIS AND DISCUSSION}

As we already mentioned above we have taken DSR routing protocol in two different environments i.e. TCP and UDP. In this we will justify in which environment DSR performs best by varying number of nodes initially we will analyze in minimum nodes then gradually we increased the number of nodes. The mobility model is used is Random waypoint mobility model because it models the random movement of the mobile nodes. Here we have used NS-2 Simulation tool to analyze the result in Linux operating system. In this NAM editor to show the animation of the communication between the nodes and $\mathrm{X}$-graph to show the graphical result of the protocol.

\subsection{Average End-To-End delay}

In this analysing study found that there is not such difference in delay in both the environment. Here we found that in TCP and UDP environment initially when we send the packet the delay is high in both the graph because initially the protocol has the discover the route to the destination so initially the delay is high then gradually it become low because at first it already generated the route and store the path details in its cache so afterward when we want to send the packet to other nodes it search the path from its cache $\mathrm{n}$ reduce the delay by skipping the route discovery process.

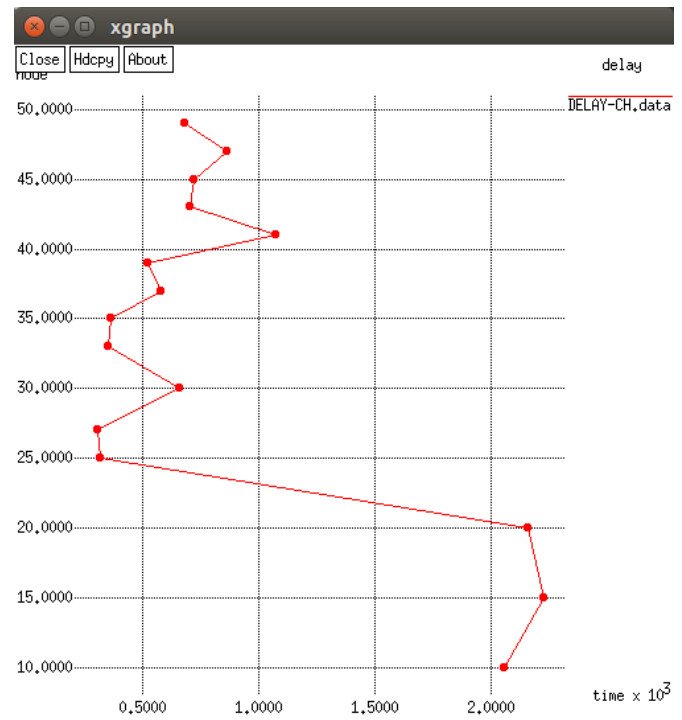

Fig 2 (a) end to end delay in UDP 


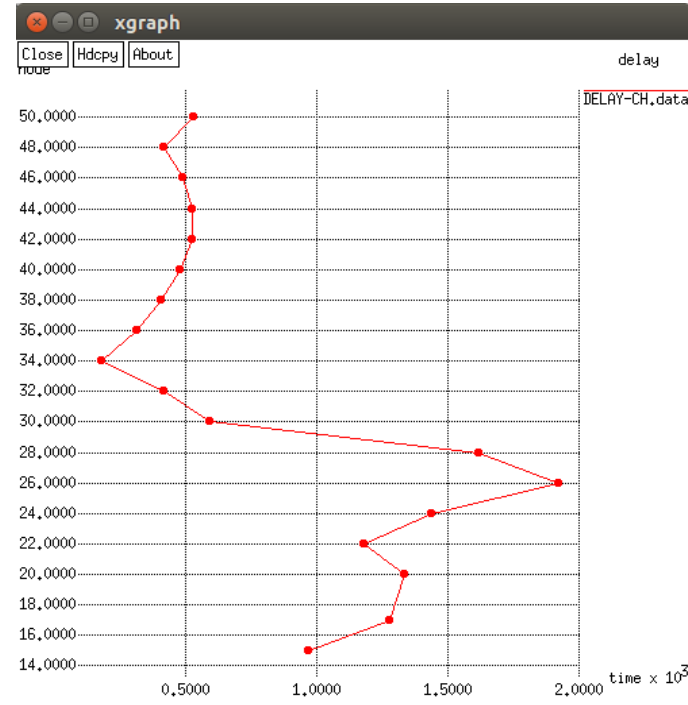

Fig 2 (b) end to end delay in TCP

\subsection{Average Throughput}

From the graph shows that DSR gives best throughput in UDP environment as compared to TCP. In UDP initially the throughput is low but when increase the number of nodes gradually the throughput goes high on the other hand in TCP environment the throughput is fluctuate. So could not say that the packets will transfer successfully while increase the number of nodes in TCP but in UDP we conform that the throughput will rise while increase the number of nodes.

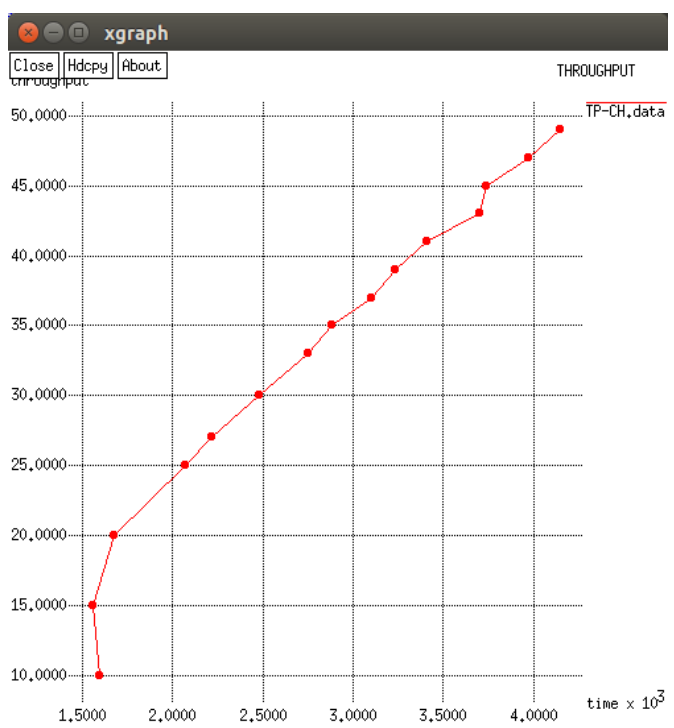

Fig 3 (a) Throughput in UDP

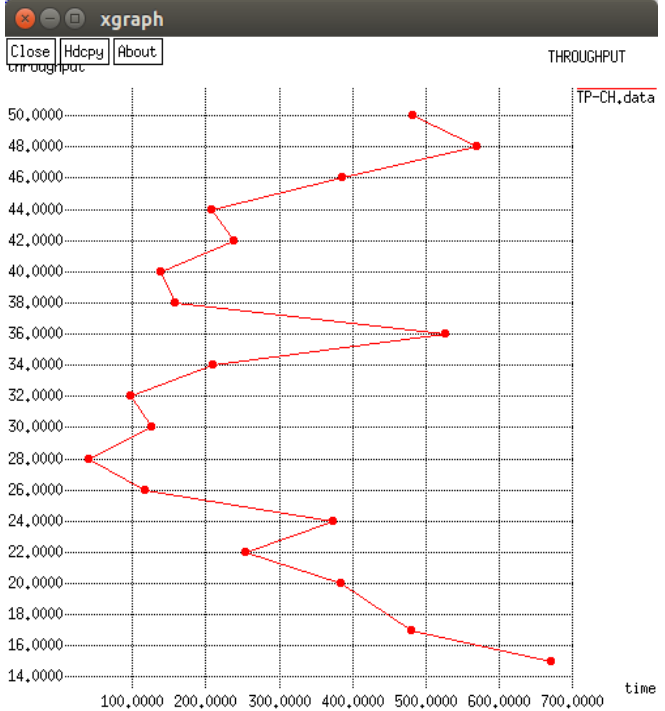

Fig 3 (b) Throughput in TCP

\subsection{Data Packet Delivery Ratio}

Here from the following graph we determine that the PDR of DSR routing protocol in UDP environment is that initially when we place minimum number of node the PDR is high but gradually it decreases because of congestions, collisions, packet loss. If we place maximum nodes the path from source to destination may varies. It get maximum path, link breakage, packets will transfer via multiple nodes. Hence the PDR gets low while increase the number of nodes on the other hand the PDR of DSR in TCP fluctuates. Initially the PDR is high then after increasing nodes it gets low and again it gets high.

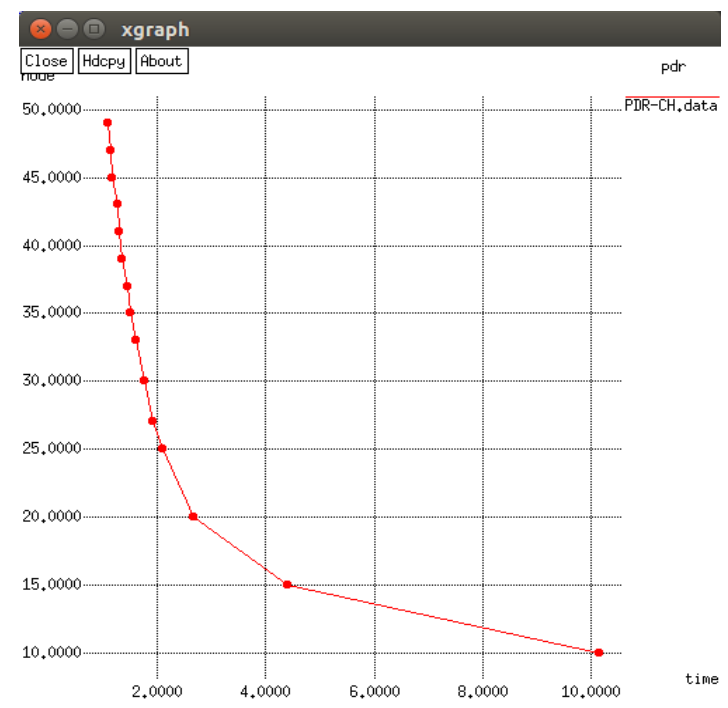

Fig 4 (a) PDR in UDP 


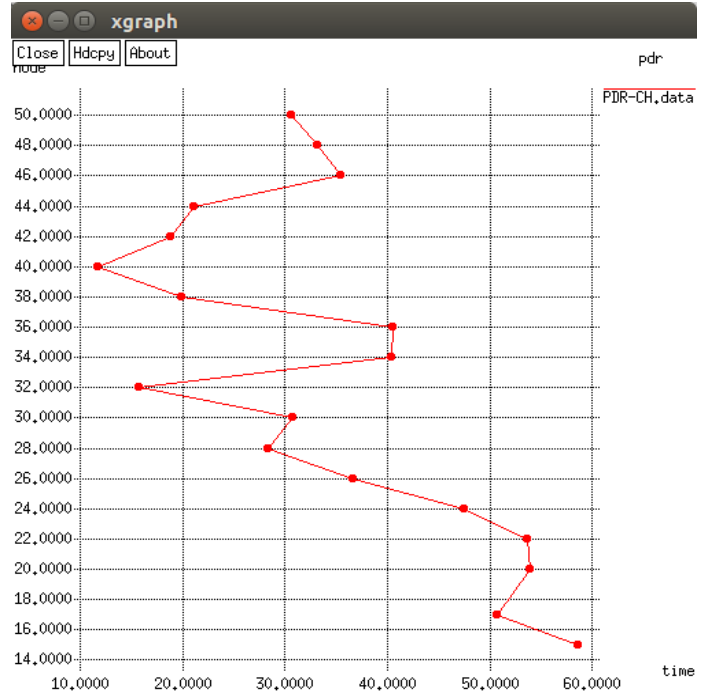

Fig 4 (b) PDR in TCP

\section{CONCLUSION}

In this paper we focused on the analysis and performance of Dynamic Source Routing (DSR) protocol with respect to TCP and UDP environment. The simulation is carried out using NS-2/RAM 1GB/500GB HD.

We have analyzed in three different scenarios i.e. PDR, Endto-End Delay and Average Throughput with respect to TCP and UDP environment by varying number of nodes. In this we found that in different environments the result of three parameters varies. In TCP environment the Throughput, Endto End Delay and Packet delivery ratio fluctuates, we could not say that at certain number of nodes we got constant good result for all the parameters. Sometimes the result of all the parameters was good and sometimes we got poor results. Whereas, in UDP environment when we analyze the performance of DSR we got good result as compared to TCP environment. In this initially in less number of nodes throughput of DSR is low but gradually when we increase the number of nodes we found that the throughput is getting high in a uniform level. The PDR in UDP environment is high initially because the number of packets is low since we increase the number of nodes gradually the PDR is low, this is because while there is maximum nodes the congestion between the nodes gets high, packet loss will high because when we send packets it may transfer via multiple nodes so it may cause maximum packet loss. On the other hand the Endto-End Delay, there is not much difference between TCP and UDP environment. In this initially the delay is high because nodes has to discover the path to send the packets to the destination but once it discover the path is stores the path in the cache of DSR so afterwards need not discover the path again. Hence the Delay gets low gradually by increasing the number of nodes.

Overall we found that DSR protocol is best suits in UDP environment as compare to TCP. The Throughput of DSR in UDP environment is good and it increases uniformly while increasing the number of nodes gradually.

\section{REFERENCES}

[1] Amit N. Thakare, Mrs. M.Y Joshi- Performance analysis of AODV \& DSR routing protocol in Mobile Ad-hoc Networks-IJCA Special on "Mobile Ad-hoc Networks" MANETs, 2000

[2] Salman Bhimla, Neeru Yadav-Comparison between AODV protocol and DSR protocol in MANET-IJAERS dec 2014

[3] Amer O. Abu Salem, Ghassan Samara, Tareq AlhmiedatPerformance analysis of Dynamic source routing protocol-JETCIS,ISSN 2079-8407 Feb 2014.

[4] Rachit jain, Laxmi Srivastava-Study and Performance Comparison of AODV \& DSR on the basic of path loss propagation models.-IJACT,july 2011.

[5] J.J Garcia-Luna-Aceves, Marc Mosko, Charles E. Perkins- A new approach to on demand loop-free routing in Ad-hoc networks-

[6] Mubashir Husain Rehmani, Sidney Doria, and Mustapha Reda Senouci-A Tutorial on the Implementation Of Adhoc on demand Distance Vector(AODV) protocol in Network Simulator(NS-2)

[7] Mobile

Ad-hoc Networks(MANET),httop://www.ietf.org/html.charters/ manetcharter.html.(1998-11-29)

[8] Hadi Sargolzaey, Ayyoub Akbari Moghanjoughi and Sabira Khatun,-A Review and Comparison of Reliable Unicast Routing Protocols For Mobile Ad-hoc Networks,IJCSNS International Journal of Computer Science and Network Security, VOL.9 No.1,pp.186196,January 2009.

[9] Zaiba Ishrat, Pankaj Singh, Rehan Ahmed- Performance Evaluation of DSDV,DSR AND ZRP Protocol in MANET-IJCATR vol 2-Issue 2,2013.

[10] Utpal barman, Neelpawan Kalita- Performance analysis of AODV routing protocol in MANET (A REVIEW)IJARCCE vol 5- Issue 1, JANUARY 2016

[11] Utpal Barman, Diganta Sahu- Performace analysis of DSDV-IJARCCE vol 5- Issue 1, JANUARY 2016 